\title{
Quasiflats with holes in reductive groups
}

\author{
KEVIN WORTMAN
}

\begin{abstract}
We give a new proof of a theorem of Kleiner-Leeb: that any quasi-isometrically embedded Euclidean space in a product of symmetric spaces and Euclidean buildings is contained in a metric neighborhood of finitely many flats, as long as the rank of the Euclidean space is not less than the rank of the target. A bound on the size of the neighborhood and on the number of flats is determined by the size of the quasi-isometry constants.

Without using asymptotic cones, our proof focuses on the intrinsic geometry of symmetric spaces and Euclidean buildings by extending the proof of Eskin-Farb's quasiflat with holes theorem for symmetric spaces with no Euclidean factors.
\end{abstract}

20F65; 20G30, 22E40

\section{Introduction}

We will give a new proof and a generalization of the following result:

Theorem 1.1 (Kleiner-Leeb) Let $\mathbb{E}^{m}$ be $m$-dimensional Euclidean space, and suppose $\varphi: \mathbb{E}^{m} \rightarrow X$ is a $(\kappa, C)$ quasi-isometric embedding, where $X$ is a product of symmetric spaces and Euclidean buildings and $m$ equals the rank of $X$. Then there exist finitely many flats $F_{1}, F_{2}, \ldots, F_{M} \subseteq X$ such that

$$
\varphi\left(\mathbb{E}^{m}\right) \subseteq \operatorname{Nbhd}_{N}\left(\bigcup_{i=1}^{M} F_{i}\right),
$$

where $M=M(\kappa, X)$ and $N=N(\kappa, C, X)$.

Theorem 1.1 was proved by Kleiner and Leeb in [5]. It can be used to give a new proof of a conjecture of Margulis from the 1970s (also proved in [5]) that any selfquasi-isometry of $X$ as above is a bounded distance from an isometry when all factors correspond to higher rank simple groups. For an indication as to how Theorem $1.1 \mathrm{can}$ be used to give a proof of this fact, see [3] where Eskin-Farb give a proof of Theorem 1.1 and Margulis' conjecture in the case when $X$ is a symmetric space. 
Our proof of Theorem 1.1 does not use asymptotic cones as the proof of KleinerLeeb does. Rather, we adapt results of Eskin-Farb who used large-scale homology to characterize quasiflats in symmetric spaces without Euclidean factors in a way that allowed for the absence of large regions in the domain of a quasiflat (a "quasiflat with holes"). Thus, we provide a marriage between the quasiflats theorems of Kleiner-Leeb and Eskin-Farb: a quasiflats theorem that allows for products of symmetric spaces and Euclidean buildings in the target of a quasiflat, and for holes in the domain; see Theorem 1.2 below. Theorem 1.1 occurs as a special case.

Allowing for holes in our quasiflats leads to applications for the study of the large-scale geometry of non-cocompact $S$-arithmetic lattices; see Wortman [9; 10].

Bibliographic note The full theorem of Kleiner-Leeb is more general than Theorem 1.1 as it allows for generalized Euclidean buildings in the target of $\varphi$. However, Theorem 1.1 does include all of the standard Euclidean buildings that are naturally acted on by reductive groups over local fields.

Quasiflats with holes For constants $\kappa \geq 1$ and $C \geq 0$, a $(\kappa, C)$ quasi-isometric embedding of a metric space $\mathrm{X}$ into a metric space $\mathrm{Y}$ is a function $\varphi: X \rightarrow Y$ such that for any $x_{1}, x_{2} \in X$ :

$$
\frac{1}{\kappa} d\left(x_{1}, x_{2}\right)-C \leq d\left(f\left(x_{1}\right), f\left(x_{2}\right)\right) \leq \kappa d\left(x_{1}, x_{2}\right)+C .
$$

For a subset of Euclidean space $\Omega \subseteq \mathbb{E}^{m}$, we let

$$
\Omega_{(\varepsilon, \rho)}=\left\{x \in \Omega \mid B_{y}(\varepsilon d(x, y)) \cap \Omega \neq \varnothing \text { for all } y \in \mathbb{E}^{m}-B_{x}(\rho)\right\},
$$

where we use the notation $B_{z}(r)$ to refer to the ball of radius $r$ centered at $z$. Hence, $\Omega_{(\varepsilon, \rho)}$ is the set of all points $x \in \Omega$ which can serve as an observation point from which all points in $\mathbb{E}^{m}$ (that are a sufficient distance from $x$ ) have a distance from $\Omega$ that is proportional to their distance from $x$.

A special case to keep in mind is that if $\Omega=\mathbb{E}^{m}$, then $\Omega_{(\varepsilon, \rho)}=\mathbb{E}^{m}$ for any $\varepsilon \geq 0$ and $\rho \geq 0$.

A quasiflat with holes is the image of $\Omega_{(\varepsilon, \rho)}$ under a quasi-isometric embedding $\phi: \Omega \rightarrow X$.

Before stating our main result, recall that for a metric space $X$, the rank of $X$ (or $\operatorname{rank}(X)$ for short) is the maximal dimension of a flat in $X$. Now we have the following generalization of Theorem 1.1: 
Theorem 1.2 (Quasiflats with holes) Let $\varphi: \Omega \rightarrow X$ be a $(\kappa, C)$ quasi-isometric embedding where $X$ is a product of symmetric spaces and Euclidean buildings, $\Omega \subseteq \mathbb{E}^{m}$, and $m \geq \operatorname{rank}(X)$. There are constants $M=M(\kappa, X)$ and $\varepsilon_{0}=\varepsilon_{0}(\kappa, X)$, such that if $\varepsilon<\varepsilon_{0}$, then there exist flats $F_{1}, F_{2}, \ldots, F_{M} \subseteq X$ such that

$$
\varphi\left(\Omega_{(\varepsilon, \rho)}\right) \subseteq \operatorname{Nbhd}_{N}\left(\bigcup_{i=1}^{M} F_{i}\right),
$$

where $N=N(\kappa, C, \rho, X)$.

Quasirank We remark that by comparing the volume of the domain and image of a function $\varphi$ satisfying the hypotheses of Theorem 1.2, it is clear that no quasi-isometric embeddings exist of a Euclidean space into $X$ when the dimension of the Euclidean space is greater than the rank of $X$. This observation is not new and follows very easily from the pre-existing quasiflats theorems. However, we choose to state our theorem in this more general manner since the proof given below does not depend on the dimension of the Euclidean space once its dimension at least equals the rank of $X$, and our proof will run more smoothly if we allow for dimensions larger than the rank of $X$.

Applications for quasiflats One would like to characterize quasiflats as a starting point for understanding quasi-isometries of a lattice as Mostow did for cocompact lattices. (See Morse [6], Mostow [7], Pansu [8], Kleiner-Leeb[5], Eskin-Farb [3], Eskin [2], Wortman [9; 10] for the details of this brief sketch.)

The basic example of a quasiflats theorem is the Morse-Mostow Lemma which states that a quasi-isometric embedding of $\mathbb{R}$ into a rank one symmetric space has its image contained in a metric neighborhood of a unique geodesic.

For general symmetric spaces and Euclidean buildings $X$, it is not the case that a quasiisometrically embedded Euclidean space is necessarily contained in the neighborhood of a single flat. (Recall that a flat is an isometrically embedded Euclidean space.) If, however, the dimension of a quasi-isometrically embedded Euclidean space is equal to the dimension of a maximal flat in $X$, then its image will be contained in a neighborhood of finitely many flats.

Quasiflats can be used in the study of quasi-isometries of cocompact lattices as follows. First, we may assume that any self-quasi-isometry of a cocompact lattice in a semisimple Lie group is a quasi-isometry of its orbit in an appropriate product of symmetric spaces and Euclidean buildings, $X$. Second, since any flat in $X$ is necessarily contained in a metric neighborhood of the cocompact lattice orbit, we can restrict the quasi-isometry 
to any flat and examine its image. The space $X$ has a boundary at infinity which is defined in terms of the asymptotic behavior of flats, so in determining the images of flats we are finding a map on the boundary of $X$. Finally-as long as $X$ contains no factors that are real hyperbolic spaces, complex hyperbolic spaces, or trees-one can deduce from the properties of the boundary map that the quasi-isometry is a finite distance from an isometry.

The story is different for non-cocompact lattices. Generic flats in $X$ will not be contained in a neighborhood of a non-cocompact lattice orbit. Hence, we cannot apply the same proof technique.

However, the generic flat will have a substantial portion of its volume contained in a neighborhood of a non-cocompact lattice orbit. With an eye towards this feature, EskinFarb provided a foundational tool for studying quasi-isometries of non-cocompact lattices in real semisimple Lie groups by defining and characterizing quasiflats with holes in symmetric spaces.

Using quasiflats with holes in symmetric spaces, Eskin developed a boundary map in the non-cocompact lattice case for real groups en route to proving that any quasi-isometry of a higher rank arithmetic group is a finite distance from a commensurator.

By allowing for Euclidean building factors in the image of a quasiflat with holes, we will be able to use this same approach to analyze quasi-isometries of non-cocompact lattices in semisimple Lie groups over arbitrary local fields.

Outline Our proof of Theorem 1.2 in the case that $X$ is a Euclidean building is self-contained aside from results of Eskin-Farb on the large-scale homology of pinched sets in Euclidean space and some consequences of those results. Hopefully, the reader who is interested in only the case when $X$ is a building can read through our proof without having to consider symmetric spaces.

In the general case, when $X$ is a nontrivial product of a symmetric space and a Euclidean building, we rely heavily on the results of Eskin-Farb for symmetric spaces. Our approach is to project the quasiflat with holes into the building factor $X_{\mathfrak{p}}$, and into the symmetric space factor $X_{\infty}$. By projecting the quasiflat with holes to $X_{\mathfrak{p}}$, we can apply arguments below that were created expressly for buildings while ignoring the symmetric space factor. Conversely, by projecting the quasiflat with holes to $X_{\infty}$, we can directly apply most of the content of [3] to analyze the image. After examining the image in each factor, we piece together the information obtained in the full space $X$ to obtain our result.

Thus, in our approach to proving Theorem 1.2, we will try to avoid dealing with the product space $X$. We do this since arguments for symmetric spaces and Euclidean 
buildings (although extremely similar in spirit) have to be dealt with using different tools.

The approach of projecting to factors is taken from the work of Eskin-Farb as well. Their test case for their general theorem was when $X=\mathbb{W}^{2} \times \mathbb{W}^{2}$, and they used the projection method to reduce most of the proof to arguments in the hyperbolic plane [4].

In Section 2 we will show that certain subspaces in $X$ which behave like rank one spaces cannot accommodate quasi-isometric embeddings of large Euclidean sets. This fact will be formulated more precisely in terms of homology.

Some of the nearly rank one spaces are then glued together to give a "degenerate space" in $X$ which is a fattening of the singular directions in $X$ with respect to a given basepoint. (Recall that a direction is singular if it is contained in more than one flat.) Using a Mayer-Vietoris sequence, it can be shown that the degenerate space cannot accommodate quasi-isometric embeddings of large Euclidean sets of large dimension. It is at this point where we apply our hypothesis that the dimension of $\mathbb{E}^{m}$ equals, or exceeds, the rank of $X$.

In Section 3 we begin to analyze the asymptotic behavior of quasiflats with holes. We define-following Eskin-Farb-what it means for a direction in a quasiflat with holes to limit on a point in the boundary at infinity of $X$.

The results of Section 2 show that the image of a quasiflat with holes must have a substantial intersection with the complement of the degenerate space. (The complement of the degenerate space is the region of $X$ for which limit points are defined.) We argue further to show that limit points exist.

Since the nondegenerate space behaves much like a rank one space itself, we can show that the image of a quasiflat with holes in the nondegenerate space cannot extend in too many directions (i.e. the number of limit points is bounded). We construct our bound by contrasting the polynomial growth of Euclidean space with the high cost of travelling out in different directions in a rank one space. It is from the finite set of limit points that the finite set of flats from the conclusion of Theorem 1.2 is constructed.

Section 4 contains a few lemmas to insure that all definitions depending on basepoints are well-defined up to a constant.

We conclude in Section 5 with a proof of Theorem 1.2. Results from Sections 2, 3 and 4 are used in the proof.

Definitions Recall that a polysimplex is a product of simplices. Replacing simplices with polysimplices in the definition of a simplicial complex creates what is called a polysimplicial complex. 
A Euclidean building $X_{\mathfrak{p}}$ is a polysimplicial complex endowed with a metric $d_{\mathfrak{p}}$ that satisfies the four properties below:

(i) There is a family, $\left\{\mathcal{A}_{\alpha}\right\}$, of subcomplexes of $X_{\mathfrak{p}}$ such that each $\mathcal{A}_{\alpha}$ is isometric to $\mathbb{E}^{\operatorname{dim}\left(X_{\mathfrak{p}}\right)}$ and $X_{\mathfrak{p}}=\bigcup_{\alpha} \mathcal{A}_{\alpha}$. Each $\mathcal{A}_{\alpha}$ is called an apartment.

(ii) Any two polysimplices of maximal dimension (called chambers) are contained in some $\mathcal{A}_{\alpha}$.

(ii) If $\mathcal{A}_{\alpha}$ and $\mathcal{A}_{\beta}$ are two apartments each containing the chambers $\mathfrak{c}_{1}$ and $\mathfrak{c}_{2}$, then there is an isometric polysimplicial automorphism of $X$ sending $\mathcal{A}_{\alpha}$ to $\mathcal{A}_{\beta}$, and fixing $\mathfrak{c}_{1}$ and $\mathfrak{c}_{2}$ pointwise.

(iv) The group of isometric polysimplicial automorphisms of $X_{\mathfrak{p}}$ acts transitively on the set of chambers.

Note that condition (iv) is nonstandard. Often one assumes the stronger condition that a building be thick. We desire to weaken the thickness condition to condition (iv) so that Euclidean space can naturally be given the structure of a Euclidean building.

Also notice that we do not assume $X_{\mathfrak{p}}$ to be locally finite. Hence, we are including the buildings for, say, $\mathbf{G L}_{\mathbf{n}}(\mathbb{C}(t))$ in our examination.

Along with the nonstandard definition of a Euclidean building given above, we also give the standard definition of a symmetric space as a Riemannian manifold $X_{\infty}$ such that for every $p \in X_{\infty}$, there is an isometry $g$ of $X_{\infty}$ such that $g(p)=p$ and the derivative of $g$ at $p$ equals - Id.

Conventions Throughout this paper we will be examining products of symmetric spaces and Euclidean buildings. Since Euclidean space is a Euclidean building by our definition, we may assume that our symmetric spaces do not have Euclidean factors. This will allow us to more readily apply results from [3] where it is assumed that the symmetric spaces have no Euclidean factors.

We may also assume that our symmetric spaces do not have compact factors. Otherwise we could simply compose the quasi-isometry $\varphi$ from Theorem 1.2 with a projection map to eliminate the compact factors, then apply Theorem 1.2, pull back the flats obtained to the entire symmetric space, and increase the size of $N$ by the diameter of the compact factors.

Notation If $a$ and $b$ are positive numbers we write $a \ll b$ when there is a constant $\lambda=\lambda(X, \kappa)<1$ such that $a<\lambda b$. If there are variables $x_{1}, \ldots, x_{n}$ and a constant $\eta=\eta\left(X, \kappa, x_{1}, \ldots, x_{n}\right)<1$ such that $a<\eta b$, then we write $a \ll_{\left(x_{1}, \ldots, x_{n}\right)} b$. We will 
use the notation $a=O(b)$ to mean that $a<\lambda b$ for some constant $\lambda=\lambda(X, \kappa)$ without specifying the size of $\lambda$.

Remarks With modification to only the conclusion of the proof of Lemma 3.6, our results hold when $\mathbb{E}^{m}$ is replaced by a 1 -connected nilpotent real Lie group. For example, this shows that a Heisenberg group cannot quasi-isometrically embed into $\mathbf{S L}_{\mathbf{4}}(k)$ for any locally compact nondiscrete field $k$.

Also the proof presented below can be modified in Lemma 3.2 to allow for the presence of $\mathbb{R}$-buildings in the target of the quasiflat with holes.

Acknowledgements Benson Farb was my PhD thesis advisor under whose direction this work was carried out. I thank him for suggesting this problem to me, and for his constant support and encouragement. Thanks also to Alex Eskin for listening to many of my ideas and for providing feedback. Thanks to Tara Brendle, Dan Margalit, Karen Vogtmann and a referee for valuable comments made on an earlier draft. I would also like to thank the University of Chicago for supporting me as a graduate student while I developed the ideas in this paper, and Cornell University for the pleasant working environment given to me while I completed the writing of this paper. I was supported in part by an NSF Postdoctoral Fellowship.

\section{Pinching functions and homology}

Throughout the remainder, let $X_{\mathfrak{p}}$ be a Euclidean building with a chosen basepoint $e_{\mathfrak{p}} \in X_{\mathfrak{p}}$, and let $X_{\infty}$ be a symmetric space with basepoint $e_{\infty} \in X_{\infty}$. We will assume that $X_{\infty}$ has no compact or Euclidean factors (see the conventions in the preceding section).

We let $X=X_{\infty} \times X_{\mathfrak{p}}$, and we define $\pi_{\infty}: X \rightarrow X_{\infty}$ and $\pi_{\mathfrak{p}}: X \rightarrow X_{\mathfrak{p}}$ to be the projection maps. Define the point $e \in X$ as the pair $\left(e_{\infty}, e_{\mathfrak{p}}\right)$.

Throughout we let $n \in \mathbb{N}$ equal $\operatorname{rank}(X)$.

Graded quasi-isometric embeddings We will put quasiflats with holes aside until the final section of this paper. We concentrate instead on embeddings of entire Euclidean spaces into $X$ under a weaker assumption than our map is a quasi-isometry.

For points $x, y_{1}, y_{2}, \ldots, y_{n} \in X$ and a number $\rho \geq 0$, we let

$$
D_{x}\left(\rho ; y_{1}, y_{2}, \ldots, y_{n}\right)=\max \left\{\rho, d\left(x, y_{1}\right), \ldots, d\left(x, y_{n}\right)\right\} .
$$


For numbers $\kappa \geq 1, \rho \geq 0$, and $\varepsilon \geq 0$, we define a function $\phi: X \rightarrow Y$ to be a $(\kappa, \rho, \varepsilon)$ graded quasi-isometric embedding based at $x \in X$ if for all $z, w \in X$ :

$$
\frac{1}{\kappa} d(z, w)-\varepsilon D_{x}(\rho ; z, w) \leq d(\phi(z), \phi(w)) \leq \kappa d(z, w)+\varepsilon D_{x}(\rho ; z, w) .
$$

A function $\phi: X \rightarrow Y$ is called $(\kappa, \rho)$ radial at $x \in X$ if for all $z \in X$ :

$$
\frac{1}{2 \kappa} D_{x}(\rho ; z) \leq d(\phi(z), \phi(x)) \leq(2 \kappa) D_{x}(\rho ; z) .
$$

Combining the two definitions above, $\phi: X \rightarrow Y$ is a $(\kappa, \rho, \varepsilon)$ radial graded quasiisometric embedding ((RGQIE) for short) based at $x$ if it is a $(\kappa, \rho, \varepsilon)$ graded quasiisometric embedding at $x$, and $\kappa$ radial at $x$.

In the proof of Theorem 1.2, we will see that one can easily extend the domain of a quasiflat with holes to all of $\mathbb{E}^{m}$ in such a way that the extension is a (RGQIE). From the behavior of (RGQIE)'s that is characterized in Sections 2 through 4, we will be able to characterize the image of a quasiflat with holes.

Until explicitly stated otherwise, let $\phi: \mathbb{E}^{m} \rightarrow X$ be a $(\kappa, \varepsilon, \rho)$ (RGQIE) based at 0 with $\phi(0)=e$. The image of such a function is a graded quasiflat.

\section{Pinching on rays in buildings Let}

$$
K=\{g \in \operatorname{Isom}(X) \mid g e=e\},
$$

and let $\gamma_{\mathfrak{p}}:[0, \infty) \rightarrow\left\{e_{\infty}\right\} \times X_{\mathfrak{p}}$ be a geodesic ray with $\gamma_{\mathfrak{p}}(0)=e$. The space $K \gamma_{\mathfrak{p}}$ is a topological tree as can be seen by restricting the geodesic retraction $X_{\mathfrak{p}} \rightarrow\left\{e_{\mathfrak{p}}\right\}$. However, the tree $K \gamma_{\mathfrak{p}}$ will often not be convex. These trees in $X$ are negatively curved, and our first goal is to show that large subsets of Euclidean space cannot embed into them, or even into small enough neighborhoods of them. This in itself is straightforward to show, but we shall want to handle this problem in a way that allows us to conclude that large Euclidean sets cannot embed into fattened neighborhoods of $K$ translates of certain $(n-1)$-dimensional spaces.

Let

$$
K \gamma_{\mathfrak{p}}(\delta)=\left\{x \in\left\{e_{\infty}\right\} \times X_{\mathfrak{p}} \mid d(x, t)<\delta d(x, e) \text { for some } t \in K \gamma_{\mathfrak{p}}\right\},
$$

so that $K \gamma_{\mathfrak{p}}(\delta)$ is a neighborhood of $K \gamma_{\mathfrak{p}}$ in $\left\{e_{\infty}\right\} \times X_{\mathfrak{p}}$ that is fattened in proportion to the distance from the origin by a factor of $\delta$. We will want to project $K \gamma_{\mathfrak{p}}(\delta)$ onto $K \gamma_{\mathfrak{p}}$ where calculations can be made more easily.

Define

$$
\pi\left(\gamma_{\mathfrak{p}}, \delta\right): K \gamma_{\mathfrak{p}}(\delta) \rightarrow K \gamma_{\mathfrak{p}}
$$

Algebraic 83 Geometric Topology, Volume 6 (2006) 
by choosing for any $x \in K \gamma_{\mathfrak{p}}(\delta)$, some $\pi\left(\gamma_{\mathfrak{p}}, \delta\right)(x) \in K \gamma_{\mathfrak{p}}$, such that

$$
d\left(x, \pi\left(\gamma_{\mathfrak{p}}, \delta\right)(x)\right) \leq \delta d(x, e) .
$$

By definition, $\pi\left(\gamma_{\mathfrak{p}}, \delta\right)$ only modifies distances by a linear error of $\delta$, so composing with $\phi$ will still be a (RGQIE). Precisely, we have the following:

Lemma 2.1 If $\varepsilon<\delta<1 / 2$, then $\pi\left(\gamma_{\mathfrak{p}}, \delta\right) \circ \phi: \phi^{-1}\left(K \gamma_{\mathfrak{p}}(\delta)\right) \rightarrow K \gamma_{\mathfrak{p}}$ is a $(2 \kappa, \rho, 5 \kappa \delta)$ (RGQIE) based at 0 .

Proof Verifying that $\pi\left(\gamma_{\mathfrak{p}}, \delta\right) \circ \phi$ is a graded quasi-isometric embedding is an easy sequence of inequalities:

$$
\begin{aligned}
d\left(\pi\left(\gamma_{\mathfrak{p}}, \delta\right) \circ \phi(x), \pi\left(\gamma_{\mathfrak{p}}, \delta\right) \circ \phi(y)\right) \\
\quad \leq d\left(\pi\left(\gamma_{\mathfrak{p}}, \delta\right) \circ \phi(x), \phi(x)\right)+d\left(\pi\left(\gamma_{\mathfrak{p}}, \delta\right) \circ \phi(y), \phi(y)\right)+d(\phi(x), \phi(y)) \\
\quad \leq d(\phi(x), \phi(y))+2 \delta D_{e}(0 ; \phi(x), \phi(y)) \\
\quad \leq \kappa d(x, y)+\varepsilon D_{0}(\rho ; x, y)+4 \kappa \delta D_{0}(\rho ; x, y) .
\end{aligned}
$$

The other inequality is similar.

That $\pi\left(\gamma_{\mathfrak{p}}, \delta\right) \circ \phi$ is radial is also straightforward:

$$
\begin{aligned}
d\left(\pi\left(\gamma_{\mathfrak{p}}, \delta\right) \circ \phi(x), e\right) & \leq d\left(\pi\left(\gamma_{\mathfrak{p}}, \delta\right) \circ \phi(x), \phi(x)\right)+d(\phi(x), e) \\
& \leq(1+\delta) d(\phi(x), e) \\
& \leq 2 \kappa(1+\delta) D_{0}(\rho ; x) .
\end{aligned}
$$

Again, the other inequality is similar.

As in [3], for numbers $r \geq 0, \eta>1$, and $\beta>0$, we define an $(r, \eta, \beta)$ pinching function on a set $W \subseteq \mathbb{E}^{m}$ to be a proper, continuous function $f: W \rightarrow \mathbb{R}_{\geq 0}$ such that for any $x, y \in W$, we have $d(x, y)<\beta s$ whenever the following two properties hold:

(i) $r \leq s \leq f(x) \leq f(y) \leq \eta s$;

(ii) there is a path $\psi:[0,1] \rightarrow W$ such that $\psi(0)=x, \psi(1)=y$, and $s \leq f(\psi(t))$ for all $t \in[0,1]$.

If there exists an $(r, \eta, \beta)$ pinching function on some $W \subseteq \mathbb{E}^{m}$, then we say that $W$ is $(r, \eta, \beta)$-pinched.

Eskin-Farb used pinching functions as a means of showing that large Euclidean sets cannot quasi-isometrically embed into certain negatively curved subspaces of symmetric spaces. To show the analogous result for our general $X$, we will first construct a 
pinching function for $\phi^{-1}\left(K \gamma_{\mathfrak{p}}(\delta)\right)$. Since Eskin-Farb constructed a pinching function on the similarly defined sets $\phi^{-1}\left(K \gamma_{\infty}(\delta)\right)$, we will then be in a position to handle the case for a general ray by pulling back pinching functions obtained through projection to factors.

Our candidate for a pinching function on $\phi^{-1}\left(K \gamma_{\mathfrak{p}}(\delta)\right)$ is

$$
f\left(\gamma_{\mathfrak{p}}, \delta\right): \phi^{-1}\left(K \gamma_{\mathfrak{p}}(\delta)\right) \rightarrow \mathbb{R}_{\geq 0}
$$

where

$$
f\left(\gamma_{\mathfrak{p}}, \delta\right)(x)=d\left(\pi\left(\gamma_{\mathfrak{p}}, \delta\right) \circ \phi(x), e\right) .
$$

Lemma 2.2 If $\varepsilon<\delta<1 / 2$, then $f\left(\gamma_{\mathfrak{p}}, \delta\right)$ is a $\left(5 \kappa \rho, 1+\delta, 84 \kappa^{3} \delta\right)$ pinching function on the set $\phi^{-1}\left(K \gamma_{\mathfrak{p}}(\delta)\right) \subseteq \mathbb{E}^{m}$.

Proof Note that we may assume $\pi\left(\gamma_{\mathfrak{p}}, \delta\right) \circ \phi$ is continuous by a connect-the-dots argument. Hence, $f\left(\gamma_{\mathfrak{p}}, \delta\right)$ is clearly continuous and proper. We assume $x, y \in$ $\phi^{-1}\left(K \gamma_{\mathfrak{p}}(\delta)\right)$ are such that

$$
5 \kappa \rho \leq s \leq f\left(\gamma_{\mathfrak{p}}, \delta\right)(x) \leq f\left(\gamma_{\mathfrak{p}}, \delta\right)(y) \leq(1+\delta) s,
$$

and there is a path $\psi:[0,1] \rightarrow \phi^{-1}\left(K \gamma_{\mathfrak{p}}(\delta)\right)$ with $s \leq f\left(\gamma_{\mathfrak{p}}, \delta\right)(\psi(t))$ for all $t \in[0,1]$.

By the radial condition of Lemma 2.1,

$$
5 \kappa \rho \leq d\left(\pi\left(\gamma_{\mathfrak{p}}, \delta\right) \circ \phi(x), e\right) \leq 4 \kappa D_{0}(\rho ; x) .
$$

It follows that $\rho<d(x, 0)$. Hence, by the radial condition of Lemma 2.1 and our pinching assumptions,

$$
d(x, 0) \leq 4 \kappa d\left(\pi\left(\gamma_{\mathfrak{p}}, \delta\right) \circ \phi(x), e\right) \leq 4 \kappa(1+\delta) s .
$$

The existence of $\psi$ implies that $\pi\left(\gamma_{\mathfrak{p}}, \delta\right) \circ \phi(x)$ and $\pi\left(\gamma_{\mathfrak{p}}, \delta\right) \circ \phi(y)$ are in the same connected component of $K \gamma_{\mathfrak{p}}-B_{e}(s)$. Therefore,

$$
d\left(\pi\left(\gamma_{\mathfrak{p}}, \delta\right) \circ \phi(x), \pi\left(\gamma_{\mathfrak{p}}, \delta\right) \circ \phi(y)\right) \leq 2 \delta s .
$$

We may assume $d(x, 0) \geq d(y, 0)$. Then, by the graded condition of Lemma 2.1,

$$
2 \delta s \geq \frac{1}{2 \kappa} d(x, y)-(5 \kappa \delta) d(x, 0) \geq \frac{1}{2 \kappa} d(x, y)-(5 \kappa \delta) 4 \kappa(1+\delta) s .
$$

That is, $d(x, y)<84 \kappa^{3} \delta s$.

Graded neighborhoods For a set $Y \subseteq X$, we can create a neighborhood of $Y$ by fattening points in $Y$ in $\delta$-proportion to their distance from $e$. In symbols, we let

$$
Y[\delta]=\{x \in X \mid d(x, y)<\delta d(x, e) \text { for some } y \in Y\} .
$$


Pinching on general rays Lemma 6.8 in [4] demonstrates a pinching function for sets of the form $\phi^{-1}\left(K \gamma_{\infty}(\delta)\right)$ where $\gamma_{\infty}:[0, \infty) \rightarrow X_{\infty} \times\left\{e_{\mathfrak{p}}\right\}$ is a geodesic ray, and $K \gamma_{\infty}(\delta) \subseteq X_{\infty} \times\left\{e_{\mathfrak{p}}\right\}$ is defined analogously to $K \gamma_{\mathfrak{p}}(\delta) \subseteq\left\{e_{\infty}\right\} \times X_{\mathfrak{p}}$. We can use this pinching function along with the pinching function from Lemma 2.2 to show that $\phi^{-1}(K \gamma[\delta])$ is a pinched set, where $\gamma:[0, \infty) \rightarrow X$ is an arbitrary geodesic ray with $\gamma(0)=e$. Our argument proceeds by simply applying our already existing pinching functions to the image of $K \gamma[\delta]$ under the projection maps onto the factors of $X$.

We want to define a real valued tilt parameter, $\tau$, on the space of geodesic rays $\gamma:[0, \infty) \rightarrow X$ with $\gamma(0)=e$. The parameter will measure whether $\gamma$ leans more towards the $X_{\mathfrak{p}}$ or the $X_{\infty}$ factor. Notice that any such $\gamma$ can be decomposed as $\gamma(t)=\left(\gamma_{\infty}(t), \gamma_{\mathfrak{p}}(a t)\right)$ for some number $a \geq 0$, and all $t \geq 0$, where $\gamma_{\infty} \subseteq X_{\infty}$ and $\gamma_{\mathfrak{p}} \subseteq X_{\mathfrak{p}}$ are unit speed geodesic rays based at $e_{\infty}$ and $e_{\mathfrak{p}}$ respectively. Now we simply set $\tau(\gamma)=a$. (For $\tau$ to be defined everywhere we allow for the case when $a=\infty$, which is just to say that $\gamma$ is contained in the building factor.) Hence, if $\tau(\gamma)>1$ (resp. $<1$ ) then $\gamma$ is leaning towards the building factor (resp. symmetric space factor), and when creating a pinching function on $K \gamma[\delta]$ it will be most efficient to project onto the $X_{\mathfrak{p}}\left(\right.$ resp. $\left.X_{\infty}\right)$ factor of $X$.

We begin with the following technical observation.

Lemma 2.3 Assume $\gamma:[0, \infty) \rightarrow X$ is a geodesic ray with $\gamma(0)=e$ and that $y \in K \gamma[\delta]$. Then,

(i) $\pi_{\mathfrak{p}}(y) \in K \gamma_{\mathfrak{p}}\left(\delta \sqrt{1+\cot ^{2}\left(\left|\tan ^{-1} \tau(\gamma)-\sin ^{-1} \delta\right|_{+}\right)}\right)$, and

(ii) $\pi_{\infty}(y) \in K \gamma_{\infty}\left(\delta \sqrt{1+\cot ^{2}\left(\left|\tan ^{-1} 1 / \tau(\gamma)-\sin ^{-1} \delta\right|_{+}\right)}\right)$,

where $|x|_{+}=\max \{x, 0\}$.

Proof By definition of $K \gamma[\delta]$ there exists a $t \geq 0$ and a $k \in K$ such that

$$
\begin{aligned}
d\left(\pi_{\mathfrak{p}}(y), k \gamma_{\mathfrak{p}}(\tau(\gamma) t)\right) & =d\left(\pi_{\mathfrak{p}}(y), \pi_{\mathfrak{p}}(k \gamma(t))\right) \\
& \leq d(y, k \gamma(t)) \\
& <\delta d(y, e) \\
& \leq \delta \sqrt{d\left(\pi_{\mathfrak{p}}(y), e_{\mathfrak{p}}\right)^{2}+d\left(\pi_{\infty}(y), e_{\infty}\right)^{2}} .
\end{aligned}
$$

Using straightforward trigonometry it can be verified that

$$
d\left(\pi_{\infty}(y), e_{\infty}\right) \leq d\left(\pi_{\mathfrak{p}}(y), e_{\mathfrak{p}}\right) \cot \left(\left|\tan ^{-1} \tau(\gamma)-\sin ^{-1} \delta\right|_{+}\right) .
$$

Then (i) follows. The proof of (ii) is similar. 
We will use part (i) of the previous lemma to create a pinching function for geodesic rays that tilt towards $X_{\mathfrak{p}}$. This is the content of Lemma 2.5, but we will first note that the projection onto $X_{\mathfrak{p}}$ does not significantly distort distances.

Lemma 2.4 Let $\gamma:[0, \infty) \rightarrow X$ be a geodesic ray with $\gamma(0)=e$. If $\varepsilon<\delta$ and $\tau(\gamma) \geq 1$, then $\pi_{\mathfrak{p}} \circ \phi: \phi^{-1}(K \gamma[\delta]) \rightarrow X_{\mathfrak{p}}$ is a $\left(2 \kappa, \rho, \eta_{1}\right)$ (RGQIE) where $\eta_{1}=O(\delta)$.

Proof Note that on $K \gamma[\delta], \pi_{\mathfrak{p}}$ is a $(2,0, O(\delta))$ (RGQIE) where 2 is an upper bound given by our restriction on $\tau(\gamma)$. Composition with $\phi$ completes the result.

Now for the pinching function:

Lemma 2.5 Let $\gamma:[0, \infty) \rightarrow X$ be a geodesic ray with $\gamma(0)=e$. For $\tau(\gamma) \geq 1$ and $\varepsilon<\delta \ll 1$, the set $\phi^{-1}(K \gamma[\delta]) \subseteq \mathbb{E}^{m}$ is $(10 \kappa \rho, 1+\delta, O(\delta))$-pinched.

Proof Let $\delta_{\mathfrak{p}}=\max \left\{2 \eta_{1}, \delta \sqrt{1+\cot ^{2}\left(\tan ^{-1} \tau(\gamma)-\sin ^{-1} \delta\right)}\right\}$, and note that our conditions on $\tau(\gamma)$ and $\delta$ imply that, say,

$$
1<\sqrt{1+\cot ^{2}\left(\tan ^{-1} \tau(\gamma)-\sin ^{-1} \delta\right)}<2 .
$$

By Lemma $2.3, \pi_{\mathfrak{p}}(K \gamma[\delta]) \subseteq K \gamma_{\mathfrak{p}}\left(\delta_{\mathfrak{p}}\right)$. Hence, we can choose our pinching function $g: \phi^{-1}(K \gamma[\delta]) \rightarrow \mathbb{R}_{\geq 0}$ to be given by

$$
g(z)=d\left(\pi\left(\gamma_{\mathfrak{p}}, \delta_{\mathfrak{p}}\right) \circ \pi_{\mathfrak{p}} \circ \phi(z), e_{\mathfrak{p}}\right) .
$$

Indeed, we can use Lemma 2.4 to replace $\phi$ with $\pi_{\mathfrak{p}} \circ \phi$ in Lemma 2.2. It follows that $g$ is a $\left(10 \kappa \rho, 1+\delta_{\mathfrak{p}}, 672 \kappa^{3} \delta_{\mathfrak{p}}\right)$ pinching function.

If $\tau(\gamma) \leq 1$, we can apply Lemma 2.3 to Lemma 6.8 of [3] and obtain a similar result. Hence, we have a pinching function on $\phi^{-1}(K \gamma[\delta])$ for any geodesic ray $\gamma$ that is based at the origin. Precisely, we have the following:

Lemma 2.6 If $\varepsilon \ll \delta \ll 1$, then the set $\phi^{-1}(K \gamma[\delta]) \subseteq \mathbb{E}^{m}$ is $\left(r_{0}, 1+O(\delta), O(\delta)\right)-$ pinched for any geodesic ray $\gamma:[0, \infty) \rightarrow X$ with $\gamma(0)=e$. Here $r_{0}=r_{0}(X, \kappa, \rho, \delta)$.

Homology results of Eskin-Farb and their consequences Pinching functions were introduced in [3] as a tool for showing that sets which simultaneously support Euclidean metrics and "quasinegatively curved" metrics must be small and, hence, cannot have any interesting large-scale homology. Precisely, we can use our Lemma 2.6 in the proof of Corollary 6.9 from [3] to show: 
Lemma 2.7 There exists a $v_{1}>0$ such that if $1 \ll_{(\rho, \delta, \varepsilon)} r$, while $\varepsilon \ll \delta \ll 1$ and $W \subseteq \phi^{-1}(K \gamma[\delta])$, then the homology of the inclusion map $\iota_{*}: H_{p}\left(W \cup B_{0}(r)\right) \rightarrow$ $H_{p}\left(W\left[v_{1} \delta\right] \cup B_{0}(r)\right)$ is zero for all $p \geq 1$.

The above lemma can be used to show, for example, that the image of $\phi$ cannot be contained in $K \gamma[\delta]$. Otherwise we could take a sphere of large radius in place of $W$ to arrive at a contradiction. This is an interesting fact, but we care to know more. We are able to use this lemma to tell us that there are much larger subspaces of $X$ that spheres cannot embed into.

The larger subspaces are defined in terms of walls, so we begin by defining the latter. A subset $H \subseteq X$ is called a wall if it is a codimension 1 affine subspace of a flat that is contained in at least two distinct flats. Note that the walls through the point $e \in X$ comprise the singular directions from $e$.

Our space $X$ resembles a rank one space, from the vantage point of $e \in X$, in the regions bounded away from the singular directions. Properties of negative curvature are a powerful tool, so we will want to show the image of $\phi$ has a substantial portion of its image bounded away from the singular directions.

It is time to define $X_{e}(\delta)$ as the $\delta$-nondegenerate space at $e \in X$ consisting of those points in $X$ that are not contained in any $\delta$-graded neighborhood of a wall containing $e$. That is

$$
X_{e}(\delta)=\bigcap_{H \in \mathcal{W}_{e}}(H[\delta])^{c},
$$

where $\mathcal{W}_{e}$ is the set of walls in $X$ that contain $e$.

The complement $X_{e}(\delta)^{c}$ of the $\delta$-nondegenerate space is the $\delta$-degenerate space. We could repeat the definition for the special case that $X$ is either a Euclidean building or a symmetric space and obtain the sets $X_{\mathfrak{p}, e_{\mathfrak{p}}}(\delta), X_{\mathfrak{p}, e_{\mathfrak{p}}}(\delta)^{c}, X_{\infty, e_{\infty}}(\delta)$, and $X_{\infty, e_{\infty}}(\delta)^{c}$.

Our goal for this section is to show that the image of $\phi$ is forced to travel in $X_{e}(\delta)$. We can use Lemma 2.7 along with a Mayer-Vietoris sequence to show that the image under $\phi$ of very large subsets of $\mathbb{E}^{m}$ indeed cannot be contained in $X_{e}(\delta)^{c}$. Note that in the Tits boundary of $X, X_{e}(\delta)^{c}$ appears as a neighborhood of the $(n-2)$-skeleton. The spaces of the form $K \gamma[\delta]$ that we considered previously appear as neighborhoods of a family of points in the Tits building. It is clear how one would want to use Lemma 2.7 and a Mayer-Vietoris argument to arrive at the following:

Lemma 2.8 There exists a constant $\nu_{2}>0$, such that if $1 \ll_{(\rho, \delta, \varepsilon)} r$ while $\varepsilon \ll \delta \ll 1$ and $W \subseteq \phi^{-1}\left(X_{e}(\delta)^{c}\right)$, then the homology of the inclusion map $\iota_{*}: H_{p}\left(W \cup B_{0}(r)\right) \rightarrow$ $H_{p}\left(W\left[v_{2} \delta\right] \cup B_{0}(r)\right)$ is zero for all $p \geq n-1$. 
The basic idea of the proof is clear but there are some technicalities to consider. This is essentially Lemma 5.6 of [3], whose proof takes place in the Tits boundary where there is no difference between symmetric spaces and buildings. Hence, the proof carries over completely to prove our Lemma 2.8 .

Unbounded, nondegenerate components of graded quasiflats Note that the above lemma tells us that large metric $(n-1)$-spheres in $\mathbb{E}^{m}$ cannot map into $X_{e}(\delta)^{c}$ under $\phi$. In Lemma 5.8 of [3], this idea is extended to show that unbounded portions of $\mathbb{E}^{m}$ map into $X_{e}(\delta)$ under $\phi$. The arguments there only involve an application of what is our Lemma 2.8 to the homology of Euclidean sets. The proof applies verbatim to yield:

Corollary 2.9 There is a constant $v_{3}>1$, such that if $\varepsilon \ll \delta \ll 1$ and $z \in \phi^{-1}\left(X_{e}(\delta)\right)$ with $1 \ll_{(\delta, \varepsilon, \rho)} r \leq d(z, 0)$, then the connected component of $\phi^{-1}\left(X_{e}\left(\delta / v_{3}\right)\right) \cap B_{0}(r)^{c}$ that contains $z$ is unbounded.

Lemma 2.8 and Corollary 2.9 are the only results from this section that will be used in the remainder of this paper. We will apply Lemma 2.8 in Section 5 during the proof of Theorem 1.2. Corollary 2.9 is used in the proof of Proposition 3.5 below to create a path in the graded quasiflat that avoids the nondegenerate space and accumulates on a point in the boundary of $X$.

\section{Limit points in Euclidean buildings}

Boundary metric A subset of a Euclidean building $\mathfrak{S} \subseteq X_{\mathfrak{p}}$ is called a sector based at $x \in X_{\mathfrak{p}}$, if it is the closure of a connected component of an apartment less all the walls containing $x$.

Let $\widehat{X}_{\mathfrak{p}}$ be the set of all sectors based at $e_{\mathfrak{p}}$. For any $\mathfrak{S} \in \widehat{X}_{\mathfrak{p}}$, let $\gamma_{\mathfrak{S}}:[0, \infty) \rightarrow \mathfrak{S}$ be the geodesic ray such that $\gamma_{\mathfrak{S}}(0)=e_{\mathfrak{p}}$, and such that $\gamma_{\mathfrak{S}}(\infty)$ is the center of mass of the boundary at infinity of $\mathfrak{S}$ with its usual spherical metric. We will also use $\gamma_{\mathfrak{S}}$ to denote the image of $\gamma_{\mathfrak{S}}:[0, \infty) \rightarrow \mathfrak{S}$.

We endow $\hat{X}_{\mathfrak{p}}$ with the metric $\hat{d}_{\mathfrak{p}}$ where

$$
\widehat{d}_{\mathfrak{p}}(\mathfrak{Y}, \mathfrak{Z})=\left\{\begin{array}{cl}
\pi, & \text { if } \gamma_{\mathfrak{Y}} \cap \gamma_{\mathfrak{Z}}=\left\{e_{\mathfrak{p}}\right\} \\
\frac{1}{\left|\gamma_{\mathfrak{Y}} \cap \gamma_{\mathfrak{Z}}\right|}, & \text { otherwise. }
\end{array}\right.
$$

In the above, $\left|\gamma_{\mathfrak{Y}} \cap \gamma_{\mathfrak{Z}}\right|$ is the length of the geodesic segment $\gamma_{\mathfrak{Y}} \cap \gamma_{\mathfrak{Z}}$. 
Note that $\widehat{d}_{\mathfrak{p}}$ is invariant under the action of the stabilizer of $e_{\mathfrak{p}}$ and is a complete ultrametric on $\hat{X}_{\mathfrak{p}}$. That $\widehat{d}_{\mathfrak{p}}$ is an ultrametric means that it is a metric, and

$$
\widehat{d}_{\mathfrak{p}}(\mathfrak{Y}, \mathfrak{Z}) \leq \max \left\{\widehat{d}_{\mathfrak{p}}(\mathfrak{Y}, \mathfrak{X}), \widehat{d}_{\mathfrak{p}}(\mathfrak{X}, \mathfrak{Z})\right\} \quad \text { for any } \mathfrak{Y}, \mathfrak{Z}, \mathfrak{X} \in \widehat{X}_{\mathfrak{p}} .
$$

We will use at times that

$$
\mathfrak{Z} \in B_{\mathfrak{S}}(r) \text { implies } B_{\mathfrak{Z}}(r)=B_{\mathfrak{S}}(r),
$$

which is a reformulation of the ultrametric property.

Measuring angles We also introduce a notion of angle between two points in a building as measured from $e_{\mathfrak{p}}$. We first define $\Phi_{\mathfrak{p}}: X_{\mathfrak{p}} \rightarrow \mathcal{P}\left(\hat{X}_{\mathfrak{p}}\right)$ by

$$
\Phi_{\mathfrak{p}}(x)=\left\{\mathfrak{S} \in \hat{X}_{\mathfrak{p}} \mid x \in \mathfrak{S}\right\},
$$

where $\mathcal{P}\left(\hat{X}_{\mathfrak{p}}\right)$ denotes the power set of $\hat{X}_{\mathfrak{p}}$.

Then for any $x, y \in X_{\mathfrak{p}}$, we define

$$
\Theta_{\mathfrak{p}}(x, y)=\inf \left\{\widehat{d}_{\mathfrak{p}}\left(\mathfrak{S}_{x}, \mathfrak{S}_{y}\right) \mid \mathfrak{S}_{x} \in \Phi_{\mathfrak{p}}(x) \text { and } \mathfrak{S}_{y} \in \Phi_{\mathfrak{p}}(y)\right\} .
$$

We think of $\Theta_{\mathfrak{p}}(x, y)$ as measuring an angle between $x$ and $y$.

We will also be measuring angles formed by triangles in a single apartment. Since apartments are Euclidean spaces, we can simply use the Euclidean measure of angle. If $\mathcal{A} \subseteq X_{\mathfrak{p}}$ is an apartment and $x, y, z \in \mathcal{A}$, we let $\measuredangle_{z}^{\mathcal{A}}(x, y)$ be the standard Euclidean angle in $\mathcal{A}$ between $x$ and $y$ as measured at $z$. For any subset $H \subseteq \mathcal{A}$, and points $x, z \in \mathcal{A}$, we let

$$
\measuredangle_{z}^{\mathcal{A}}(x, H)=\min \left\{\measuredangle_{z}^{\mathcal{A}}(x, h) \mid h \in H\right\}
$$

Core of a sector From here on we will assume that $0 \leq \delta \leq 1$. For any $\mathfrak{S} \in \hat{X}_{\mathfrak{p}}$, we let

$$
\mathfrak{S}(\delta)=\{x \in \mathfrak{S} \mid d(\partial \mathfrak{S}, x) \geq \delta d(e, x)\} .
$$

We refer to $\mathfrak{S}(\delta)$ as the $\delta$-core of $\mathfrak{S}$. Note that

$$
\bigcup_{\mathfrak{S} \in \widehat{X}_{\mathfrak{p}}} \mathfrak{S}(\delta)=X_{\mathfrak{p}, e_{\mathfrak{p}}}(\delta),
$$

where $X_{\mathfrak{p}, e_{\mathfrak{p}}}(\delta)$ is the $\delta$-nondegenerate space of $X_{\mathfrak{p}}$ at $e_{\mathfrak{p}}$.

Relations between angles and distances It is clear that geodesic rays based at $e_{\mathfrak{p}}$ and travelling into the core of a sector travel transversely to walls. We need a quantitative form of this fact which is the substance of the following: 
Lemma 3.1 Suppose $\mathfrak{S} \in \widehat{X}_{\mathfrak{p}}$ and $\mathfrak{S} \subseteq \mathcal{A}$ for some apartment $\mathcal{A}$. Assume that $x \in \mathfrak{S}(\delta), z \in \gamma_{\mathfrak{S}}$, and $H_{z} \subseteq \mathcal{A}$ is a wall containing $z$. Then

$$
\measuredangle_{z}^{\mathcal{A}}\left(x, H_{z}\right) \geq \sin ^{-1}(\delta / 2)
$$

whenever $d\left(x, e_{\mathfrak{p}}\right) \geq r$ and $d\left(z, e_{\mathfrak{p}}\right) \leq(\delta r) / 2$.

Proof Notice that $\measuredangle_{z}^{\mathcal{A}}\left(x, H_{z}\right)$ is minimized when $x \in \partial \mathfrak{S}(\delta), d\left(x, e_{\mathfrak{p}}\right)=r$, and $H_{z}$ is parallel to a wall $H_{e_{\mathfrak{p}}}$ that bounds $\mathfrak{S}$. Therefore, we will assume these three statements are true. Clearly, $\measuredangle_{z}^{\mathcal{A}}\left(x, H_{z}\right)=\measuredangle_{z}^{\mathcal{A}}\left(x, \pi_{H_{z}}(x)\right)$ where $\pi_{H_{z}}: \mathcal{A} \rightarrow H_{z}$ is the orthogonal projection.

Note that

$$
d\left(H_{z}, H_{e_{\mathfrak{p}}}\right) \leq d\left(z, e_{\mathfrak{p}}\right) \leq \frac{\delta r}{2}
$$

and

$$
d\left(x, H_{e_{\mathfrak{p}}}\right)=d(x, \partial \mathfrak{S})=\delta r .
$$

Therefore,

$$
d\left(x, \pi_{H_{z}}(x)\right)=d\left(x, H_{e_{\mathfrak{p}}}\right)-d\left(H_{e_{\mathfrak{p}}}, H_{z}\right) \geq \delta r-\frac{\delta r}{2}=\frac{\delta r}{2} .
$$

We conclude the proof by observing that

$$
\measuredangle_{z}^{\mathcal{A}}\left(x, \pi_{H_{z}}(x)\right)=\sin ^{-1}\left[\frac{d\left(x, \pi_{H_{z}}(x)\right)}{d(x, z)}\right] \geq \sin ^{-1}(\delta / 2)
$$

since $d(x, z) \leq d\left(x, e_{\mathfrak{p}}\right) \leq r$.

The next lemma shows that deep points in the nondegenerate region of $X_{\mathfrak{p}}$ at $e_{\mathfrak{p}}$ that are separated by a large angle measured at $e_{\mathfrak{p}}$ must be a large distance apart. A form of notation we will use in the proof is $\left[e_{\mathfrak{p}}, z\right]$ to denote the geodesic segment with endpoints at $e_{\mathfrak{p}}$ and $z$.

Lemma 3.2 Suppose $x, y \in X_{\mathfrak{p}, e_{\mathfrak{p}}}(\delta)$ and $\Theta_{\mathfrak{p}}(x, y) \geq 2 /(\delta r)$, while $d\left(x, e_{\mathfrak{p}}\right) \geq r$ and $d\left(y, e_{\mathfrak{p}}\right) \geq r$. Then $d(x, y) \geq(\delta r) / 2$ as long as $\delta \leq 1$.

Proof Choose sectors $\mathfrak{S}_{x}, \mathfrak{S}_{y} \in \hat{X}_{\mathfrak{p}}$ such that $\mathfrak{S}_{x} \in \Phi_{\mathfrak{p}}(x)$ and $\mathfrak{S}_{y} \in \Phi_{\mathfrak{p}}(y)$. Let $z \in X_{\mathfrak{p}}$ be such that $\gamma_{x} \cap \gamma_{y}=\left[e_{\mathfrak{p}}, z\right]$. Then, we have $d\left(e_{\mathfrak{p}}, z\right) \leq(\delta r) / 2$ since $\widehat{d}_{\mathfrak{p}}\left(\mathfrak{S}_{x}, \mathfrak{S}_{y}\right) \geq 2 /(\delta r)$.

Choose an apartment $\mathcal{A}_{x}$ containing $\mathfrak{S}_{x}$. Note that $\mathfrak{S}_{y} \cap \mathcal{A}_{x}$ is a convex polyhedron $P$ in $\mathcal{A}_{x}$ that is bounded by walls. Since $z \in \partial P$, there must be a wall $H_{z} \subseteq \mathcal{A}_{x}$ such that $z \in H_{z}$ and $\mathcal{A}_{x}-H_{z}$ has a component which does not intersect $\mathfrak{S}_{y}$. Choose a 
chamber $\mathfrak{c}_{z} \subseteq \mathfrak{S}_{x}$ containing $z$ whose interior lies in this component, and such that $F=\mathfrak{c}_{z} \cap \mathcal{A}_{y}$ is a codimension 1 simplex in $\mathfrak{c}_{z}$.

Let $\mathfrak{c}_{y} \subseteq \mathfrak{S}_{y}$ be a chamber containing $y$. Note that $[z, y] \cup \mathfrak{c}_{z} \subseteq \mathcal{B}\left(\mathfrak{c}_{z}, \mathfrak{c}_{y}\right)$, where $\mathcal{B}\left(\mathfrak{c}_{z}, \mathfrak{c}_{y}\right)$ is the union of minimal galleries from $\mathfrak{c}_{z}$ to $\mathfrak{c}_{y}$. Hence, $[z, y] \cup \mathfrak{c}_{z}$ is contained in an apartment (see e.g. [1] VI.6). Therefore, $\left.\varrho\left(\mathcal{A}_{x}, \mathfrak{c}_{z}\right)\right|_{\mathcal{B}\left(\mathfrak{c}_{z}, \mathfrak{c}_{y}\right)}$ is an isometry, where $\varrho\left(\mathcal{A}_{x}, \mathfrak{c}_{z}\right): X_{\mathfrak{p}} \rightarrow \mathcal{A}_{x}$ is the building retraction corresponding to the pair $\left(\mathcal{A}_{x}, \mathfrak{c}_{z}\right)$.

Since $F \subseteq \mathcal{A}_{y}$, there is a unique wall $H_{z}^{\prime} \subseteq \mathcal{A}_{y}$ containing $F$. Since $F \subseteq H_{z}$ as well, we have $\measuredangle_{z}^{\mathcal{A}_{y}}\left(y, H_{z}^{\prime}\right)=\measuredangle_{z}^{\mathcal{A}_{x}}\left(\varrho\left(\mathcal{A}_{x}, \mathfrak{c}_{z}\right)(y), H_{z}\right)$.

Since $\varrho\left(\mathcal{A}_{x}, \mathfrak{c}\right)$ is distance decreasing, and since $H_{z}$ separates $x$ from $\varrho\left(\mathcal{A}_{x}, \mathfrak{c}_{z}\right)(y)$, we have using Lemma 3.1:

$$
\begin{aligned}
d(x, y) & \geq d\left(\varrho\left(\mathcal{A}_{x}, \mathfrak{c}_{z}\right)(x), \varrho\left(\mathcal{A}_{x}, \mathfrak{c}_{z}\right)(y)\right) \\
& =d\left(x, \varrho\left(\mathcal{A}_{x}, \mathfrak{c}_{z}\right)(y)\right) \\
& \geq d\left(x, H_{z}\right)+d\left(\varrho\left(\mathcal{A}_{x}, \mathfrak{c}_{z}\right)(y), H_{z}\right) \\
& =\sin \left[\measuredangle_{z}^{\mathcal{A}_{x}}\left(x, H_{z}\right)\right] d(z, x)+\sin \left[\measuredangle_{z}^{\mathcal{A}_{x}}\left(\varrho\left(\mathcal{A}_{x}, \mathfrak{c}_{z}\right)(y), H_{z}\right)\right] d\left(z, \varrho\left(\mathcal{A}_{x}, \mathfrak{c}_{z}\right)(y)\right) \\
& =\sin \left[\measuredangle_{z}^{\mathcal{A}_{x}}\left(x, H_{z}\right)\right] d(z, x)+\sin \left[\measuredangle_{z}^{\mathcal{A}_{y}}\left(y, H_{z}^{\prime}\right)\right] d(z, y) \\
& \geq \frac{\delta}{2}\left(d\left(x, e_{\mathfrak{p}}\right)-d\left(e_{\mathfrak{p}}, z\right)\right)+\frac{\delta}{2}\left(d\left(y, e_{\mathfrak{p}}\right)-d\left(e_{\mathfrak{p}}, z\right)\right) \\
& \geq \delta r\left(1-\frac{\delta}{2}\right) \\
& \geq \frac{\delta r}{2}
\end{aligned}
$$

Our next lemma states that, after deleting a large compact set, if the core of two sectors based at $e_{\mathfrak{p}}$ have a nontrivial intersection, then the two sectors are close in the boundary metric.

Lemma 3.3 Let $\mathfrak{S}_{1}, \mathfrak{S}_{2} \in \widehat{X}_{\mathfrak{p}}$, and suppose that $\mathfrak{S}_{1}(\delta) \cap \mathfrak{S}_{2}(\delta) \cap B_{e_{\mathfrak{p}}}(r)^{c} \neq \varnothing$. Then $\widehat{d}_{\mathfrak{p}}\left(\mathfrak{S}_{1}, \mathfrak{S}_{2}\right) \leq 2 /(\delta r)$.

Proof We prove the contrapositive. That is, we assume that $\gamma_{\mathfrak{S}_{1}} \cap \gamma_{\mathfrak{S}_{2}}=\left[e_{\mathfrak{p}}, z\right]$ where $d\left(e_{\mathfrak{p}}, z\right)<(\delta r) / 2$.

Choose an apartment $\mathcal{A}$ with $\mathfrak{S}_{2} \subseteq \mathcal{A}$. We pick a wall, $H_{z}$, with $z \in H_{z} \subseteq \mathcal{A}$ and such that $\mathfrak{S}_{1} \cap \mathfrak{S}_{2} \subseteq \bar{J}$, where $J$ is a component of $\mathcal{A}-H_{z}$ and $\bar{J}$ is the closure of $J$. 
By Lemma 3.1, $x \in \mathfrak{S}_{2}(\delta) \cap B_{e}(r)^{c}$ implies that $\measuredangle_{z}^{\mathcal{A}}\left(x, H_{z}\right) \geq \sin ^{-1}(\delta / 2)$. Hence, any such $x$ must be bounded away from $H_{z}$ and, thus, from $\bar{J}$. We have shown

$$
\mathfrak{S}_{1}(\delta) \cap \mathfrak{S}_{2}(\delta) \cap B_{e}(r)^{c} \subseteq \bar{J} \cap \mathfrak{S}_{2}(\delta) \cap B_{e}(r)^{c}=\varnothing
$$

as desired.

To travel in the nondegenerate space between two deep points separated by a large angle, one must pass near the origin. More precisely we have the following:

Lemma 3.4 (No shifting) Suppose there is a path $c:[0,1] \rightarrow X_{e}(\delta) \cap B_{e}(r)^{c}$. Then $\Theta_{\mathfrak{p}}(c(0), c(1)) \leq 2 /(\delta r)$.

Proof Since $[0,1]$ is compact, it is contained in finitely many sectors $\mathfrak{S}_{0}, \mathfrak{S}_{1}, \ldots, \mathfrak{S}_{k}$ $\in \hat{X}_{\mathfrak{p}}$. We may assume that these sectors are ordered so that there exists a partition of $[0,1]$ of the form $0=t_{0}<t_{1}<\ldots<t_{k}=1$ with $c(0) \in \mathfrak{S}_{0}, c(1) \in \mathfrak{S}_{k}$, and $c\left[t_{i}, t_{i+1}\right] \subseteq \mathfrak{S}_{i}$.

Notice that our partition requires that $c\left(t_{i}\right) \in \mathfrak{S}_{i} \cap \mathfrak{S}_{i+1}$. Hence, we can apply Lemma 3.3 to obtain that $\widehat{d}_{\mathfrak{p}}\left(\mathfrak{S}_{i}, \mathfrak{S}_{i+1}\right) \leq 2 /(\delta r)$ for all $i$. Therefore,

$$
\Theta_{\mathfrak{p}}(c(0), c(1)) \leq \widehat{d}_{\mathfrak{p}}\left(\mathfrak{S}_{0}, \mathfrak{S}_{k}\right) \leq \max \left\{\widehat{d}_{\mathfrak{p}}\left(\mathfrak{S}_{i}, \mathfrak{S}_{i+1}\right)\right\} \leq \frac{2}{\delta r} .
$$

Limit points Let $\hat{X}_{\infty}$ be the Furstenberg boundary of $X_{\infty}$. That is, we let $\hat{X}_{\infty}$ be the space of all Weyl chambers up to Hausdorff equivalence. We endow $\hat{X}_{\infty}$ with the standard metric, $\hat{d}_{\infty}$, invariant under the stabilizer of $e_{\infty}$. We let $\Phi_{\infty}: X_{\infty, e_{\infty}}(\delta) \rightarrow$ $\hat{X}_{\infty}$ be the function that sends a point to its image at infinity. As $X$ is the product of $X_{\infty}$ and $X_{\mathfrak{p}}$, we define $\hat{X}=\hat{X}_{\infty} \times \hat{X}_{\mathfrak{p}}$.

A $\delta$-limit point of $\phi$ from $e$ is a boundary point $(\mathfrak{C}, \mathfrak{S}) \in \hat{X}$, such that there exists a path $\psi:[0, \infty) \rightarrow \phi^{-1}\left(X_{e}(\delta)\right)$ that escapes every compact set, $\lim _{t \rightarrow \infty} \Phi_{\infty} \circ \phi \circ \psi(t)=\mathfrak{C}$, and $\lim _{t \rightarrow \infty} \Phi_{\mathfrak{p}} \circ \phi \circ \psi(t)=\{\mathfrak{S}\}$. If this is the case we call $\psi$ a limit path from $e$, and we write that $\psi$ limits to $(\mathfrak{C}, \mathfrak{S})$. We call the set of all $\delta$ limit points of $\phi$ from $e$, the $\delta$-limit set of $\phi$ from $e$. We denote the $\delta$-limit set of $\phi$ from $e$ by $\mathcal{L}_{\phi, e}(\delta)$.

Existence of nondegenerate visual directions For the next result of this section, we return to the material of Section 2 and in particular to Corollary 2.9.

Later we will want to show there are a finite number of limit points in the limit set of $\phi$ to create the finite number of flats for the conclusion of Theorem 1.2. This plan will only succeed if there is a limit point to start with. The results of Section 2 were derived for the purpose of showing that limit points exist. By the Proposition below, we not only know they exist, we also have precise information on how to construct them. 
Proposition 3.5 (Deep points extended to limit points) Let $v_{3}$ be as in Corollary 2.9. There is a constant $\eta_{2}=\eta_{2}(\kappa, \delta)$, such that if $\varepsilon \ll \delta \ll 1$ and $z \in \phi^{-1}\left(X_{e}(\delta)\right)$ with $1 \ll_{(\delta, \varepsilon, \rho)} r \leq d(z, 0)$, then there exists a boundary point $(\mathfrak{C}, \mathfrak{S}) \in \mathcal{L}_{\phi, e}\left(\delta / v_{3}\right)$, such that

and

$$
\widehat{d}_{\mathfrak{p}}\left(\mathfrak{S}, \Phi_{\mathfrak{p}} \circ \phi_{\mathfrak{p}}(z)\right) \leq \frac{2}{\delta r}
$$

$$
\widehat{d}_{\infty}\left(\mathfrak{C}, \Phi_{\infty} \circ \phi_{\infty}(z)\right) \leq e^{-\eta_{2} r}
$$

Proof Let $U$ be the connected component of $\phi^{-1}\left(X_{e}\left(\delta / v_{3}\right)\right) \cap B_{0}(r)^{c}$ that contains $z$. From Corollary 2.9 we know that $U$ is unbounded, so there exists a path $\psi:[0, \infty) \rightarrow U$ with $\psi(0)=z$ and such that $\psi$ escapes every compact set.

Applying Lemma 3.4, we have that the diameter of $\Phi_{\mathfrak{p}} \circ \phi_{\mathfrak{p}} \circ \psi([s, \infty))$ is at most $2 /\left(\delta R_{S}\right)$, where $R_{s}=d(0, \psi([s, \infty)))$. Notice that $R_{s} \rightarrow \infty$ as $s \rightarrow \infty$, and

$$
\Phi_{\mathfrak{p}} \circ \phi_{\mathfrak{p}} \circ \psi([t, \infty)) \subseteq \Phi_{\mathfrak{p}} \circ \phi_{\mathfrak{p}} \circ \psi([s, \infty))
$$

when $0 \leq s \leq t$. Therefore, $\lim _{s \rightarrow \infty} \Phi_{\mathfrak{p}} \circ \phi_{\mathfrak{p}} \circ \psi(s)$ exists. Call this limit $\{\mathfrak{S}\}$.

We conclude by remarking that $\widehat{d}_{\mathfrak{p}}\left(\mathfrak{S}, \Phi_{\mathfrak{p}} \circ \phi_{\mathfrak{p}}(z)\right) \leq 2 /(\delta r)$ since

$$
\Phi_{\mathfrak{p}} \circ \phi_{\mathfrak{p}}(z)=\Phi_{\mathfrak{p}} \circ \phi_{\mathfrak{p}} \circ \psi(0) \in \Phi_{\mathfrak{p}} \circ \phi_{\mathfrak{p}} \circ \psi([0, \infty))
$$

and $R_{0}=r$.

The second part of the proposition is the content of Proposition 5.9 from [3].

A bound on visual directions for annuli Once we show that there is a bound on the number of directions at infinity that a graded quasiflat can extend in, we can produce a finite collection of flats that will be our candidates for satisfying the conclusion of Theorem 1.2.

Before showing that the number of asymptotic directions a graded quasiflat travels in is bounded, we will show that the number of directions is bounded for a quasi-annuli. This bound is independent of the size of the quasi-annuli. We will then be in a position to apply the no shifting Lemma in a limiting argument to show that the same bound exists for the number of directions of a graded quasiflat.

Let $A_{R} \subseteq X_{\mathfrak{p}}$ be the annulus centered at $e_{\mathfrak{p}}$, with inner radius $R$ and outer radius $2 R$. Let $\phi_{\infty}=\pi_{\infty} \circ \phi$, and let $\phi_{\mathfrak{p}}=\pi_{\mathfrak{p}} \circ \phi$.

Before proceeding, note that $\pi_{\infty}\left(X_{e}(\delta)\right)=X_{\infty, e_{\infty}}(\delta)$ and $\pi_{\mathfrak{p}}\left(X_{e}(\delta)\right)=X_{\mathfrak{p}, e_{\mathfrak{p}}}(\delta)$. 
Lemma 3.6 The image of $\pi_{\mathfrak{p}}\left[\phi\left(A_{R}\right) \cap X_{e}(\delta)\right]$ under $\Phi_{\mathfrak{p}}$ can be covered by $c_{\mathfrak{p}}=$ $O\left(1 / \delta^{2 m}\right)$ disjoint balls of radius $(4 \kappa) /\left(\delta^{2} R\right)$ for $R>\rho$ and $\varepsilon \ll \delta$.

Proof Let $\mathfrak{S}_{i} \in \hat{X}_{\mathfrak{p}}$ be such that $\cup_{i} B_{\mathfrak{S}_{i}}\left(\frac{4 \kappa}{\delta^{2} R}\right)=\hat{X}_{\mathfrak{p}}$, and $B_{\mathfrak{S}_{i}}\left(\frac{4 \kappa}{\delta^{2} R}\right) \cap B_{\mathfrak{S}_{j}}\left(\frac{4 \kappa}{\delta^{2} R}\right)=\varnothing$ if $i \neq j$. That the balls can be chosen to be disjoint is a consequence of the ultrametric property for $\hat{X}_{\mathfrak{p}}$.

We will twice make use of the fact that if $x \in A_{R} \cap \phi^{-1}\left(X_{e}(\delta)\right)$, then

$$
\begin{aligned}
d\left(\phi_{\mathfrak{p}}(x), e_{\mathfrak{p}}\right) & =d\left(\phi(x),\left(\phi_{\infty}(x), e_{\mathfrak{p}}\right)\right) \\
& \geq \delta d(\phi(x), e) \\
& \geq \frac{\delta}{2 \kappa} D_{0}(\rho ; x) \\
& \geq \frac{\delta R}{2 \kappa}
\end{aligned}
$$

We claim that for any $x \in A_{R} \cap \phi^{-1}\left(X_{e}(\delta)\right)$,

$$
\Phi_{\mathfrak{p}}\left(\phi_{\mathfrak{p}}(x)\right) \subseteq B_{\mathfrak{S}_{i}}\left(\frac{4 \kappa}{\delta^{2} R}\right) \text { for some } i .
$$

Indeed, suppose $\mathfrak{Z}, \mathfrak{Y} \in \Phi_{\mathfrak{p}}\left(\phi_{\mathfrak{p}}(x)\right)$, and that $\mathfrak{Z} \in B_{\mathfrak{S}_{i}}\left(\frac{4 \kappa}{\delta^{2} R}\right)$. Notice that $\phi_{\mathfrak{p}}(x) \in$ $X_{\mathfrak{p}, e_{\mathfrak{p}}}(\delta)$, so we can apply (1) and Lemma 3.3 to obtain

$$
\widehat{d}_{\mathfrak{p}}(\mathfrak{Z}, \mathfrak{Y}) \leq \frac{4 \kappa}{\delta^{2} R}
$$

Therefore,

$$
\widehat{d}_{\mathfrak{p}}\left(\mathfrak{Y}, \mathfrak{S}_{i}\right) \leq \max \left\{\widehat{d}_{\mathfrak{p}}(\mathfrak{Y}, \mathfrak{Z}), \widehat{d}_{\mathfrak{p}}\left(\mathfrak{Z}, \mathfrak{S}_{i}\right)\right\} \leq \frac{4 \kappa}{\delta^{2} R}
$$

as claimed.

Suppose $i \neq j$. If $\Phi_{\mathfrak{p}}\left(\phi_{\mathfrak{p}}(x)\right) \subseteq B_{\mathfrak{S}_{i}}\left(\frac{4 \kappa}{\delta^{2} R}\right)$ and $\Phi_{\mathfrak{p}}\left(\phi_{\mathfrak{p}}(y)\right) \subseteq B_{\mathfrak{S}_{j}}\left(\frac{4 \kappa}{\delta^{2} R}\right)$ for a pair of points $x, y \in A_{R} \cap \phi^{-1}\left(X_{e}(\delta)\right)$, then $B_{\mathfrak{S}_{i}}\left(\frac{4 \kappa}{\delta^{2} R}\right) \cap B_{\mathfrak{S}_{j}}\left(\frac{4 \kappa}{\delta^{2} R}\right)=\varnothing$. Hence, by the ultrametric property of $\hat{X}_{\mathfrak{p}}$ we have

$$
\widehat{d}_{\mathfrak{p}}\left(\Phi_{\mathfrak{p}} \circ \phi_{\mathfrak{p}}(x), \Phi_{\mathfrak{p}} \circ \phi_{\mathfrak{p}}(y)\right) \geq \frac{4 \kappa}{\delta^{2} R}=\frac{2}{\delta(\delta R / 2 \kappa)} .
$$

Therefore,

$$
d\left(\phi_{\mathfrak{p}}(x), \phi_{\mathfrak{p}}(y)\right) \geq \frac{\delta(\delta R / 2 \kappa)}{2}=\frac{\delta^{2} R}{4 \kappa}
$$


by (1) and Lemma 3.3. Thus,

$$
\begin{aligned}
d(x, y) & \geq \frac{1}{\kappa} d(\phi(x), \phi(y))-\varepsilon D_{0}(\rho ; x, y) \\
& \geq \frac{1}{\kappa} d\left(\phi_{\mathfrak{p}}(x), \phi_{\mathfrak{p}}(y)\right)-\varepsilon D_{0}(\rho ; x, y) \\
& \geq \frac{\delta^{2} R}{4 \kappa^{2}}-\varepsilon 2 R \\
& \geq \frac{\delta^{2} R}{5 \kappa^{2}}
\end{aligned}
$$

In summary, we have shown that

$$
d\left(B_{i}, B_{j}\right) \geq \frac{\delta^{2} R}{5 \kappa^{2}} \quad(i \neq j)
$$

where

$$
B_{i}=A_{R} \cap \phi^{-1}\left[\pi_{\mathfrak{p}}^{-1}\left[\Phi_{\mathfrak{p}}^{-1}\left[B_{\mathfrak{S}_{i}}\left(\frac{4 \kappa}{\delta^{2} R}\right)\right]\right] \cap X_{e}(\delta)\right] .
$$

If $\mu_{m}$ is Lebesgue measure on $\mathbb{E}^{m}$, then

$$
\mu_{m}\left[A_{R} \cap \phi^{-1}\left(X_{e}(\delta)\right)\right] \leq \mu_{m}\left[A_{R}\right]<\mu_{m}\left[B_{0}(1)\right](2 R)^{m} .
$$

Combining (2) and (3) tells us that the number of nonempty $B_{i}$ is bounded above by

$$
\frac{\left(10 \kappa^{2}\right)^{m}(2 R)^{m}}{\left(\delta^{2} R\right)^{m}}=\frac{20^{m} \kappa^{2 m}}{\delta^{2 m}}
$$

We will also need to know that projecting onto the symmetric space factor will produce a bound on the visual angles there. This is Lemma 4.2 in [3] which we state as

Lemma 3.7 There exists a constant $\eta_{3}=\eta_{3}(\kappa, \delta)$, such that the image of $\pi_{\infty}\left[\phi\left(A_{R}\right) \cap X_{e}(\delta)\right]$ under $\Phi_{\infty}$ can be covered by $c_{\infty}=O\left(1 / \delta^{2 m}\right)$ balls of radius $e^{-\eta_{3} R}$ for $1 \ll{ }_{(\rho, \delta)} R$ and $\varepsilon \ll \delta$.

Note that in [3] there is no building factor. Thus, the statement of Lemma 4.2 in [3] does not mention the projection map $\pi_{\infty}$. Also note that the number of balls in [3] Lemma 4.2 is bounded by the smaller term $O\left(1 / \delta^{m}\right)$. When projecting, a factor of $\delta$ makes its way into the proof from the inequality $d\left(\pi_{\infty}(x), e_{\infty}\right) \geq \delta d(x, e)$ for $x \in X_{e}(\delta)$. The extra factor of $\delta$ influences $c_{\infty}$ by adjusting the bound from $O\left(1 / \delta^{m}\right)$ to $O\left(1 / \delta^{2 m}\right)$, and our constant $\eta_{3}$ is proportional to the corresponding constant in [3]. Aside from these minor adjustments, the proof carries through without modification.

Algebraic 83 Geometric Topology, Volume 6 (2006) 
A bound on visual directions for entire quasiflats Using the bound on the number of visual directions for annuli, we are prepared to pass to the limit and produce a bound for the number of $\delta$-limit points of $\phi$.

Proposition 3.8 (Finite limit set) For $\delta$ sufficiently small, $\left|\mathcal{L}_{\phi, e}(\delta)\right|<c_{\infty} c_{\mathfrak{p}}$.

Proof Assume there are $c_{\infty} c_{\mathfrak{p}}+1$ limit points $\left\{\left(\mathfrak{C}_{i}, \mathfrak{S}_{i}\right)\right\}_{i=1}^{c_{\infty} c_{\mathfrak{p}}+1}$. We will arrive at a contradiction.

There are two cases to consider as either

$$
\left|\left\{\mathfrak{C}_{i}\right\}_{i=1}^{c_{\infty} c_{\mathfrak{p}}+1}\right|>c_{\infty} \quad \text { or } \quad\left|\left\{\mathfrak{S}_{i}\right\}_{i=1}^{c_{\infty} c_{\mathfrak{p}}+1}\right|>c_{\mathfrak{p}}
$$

We will begin by assuming the latter.

After possibly re-indexing, let $\mathfrak{S}_{1}, \mathfrak{S}_{2}, \ldots \mathfrak{S}_{c_{\mathfrak{p}+1}}$ be distinct elements of $\left\{\mathfrak{S}_{i}\right\}_{i=1}^{c_{\infty} c_{\mathfrak{p}}+1}$. Let $\alpha=\min _{i \neq j}\left\{\hat{d}\left(\mathfrak{S}_{i}, \mathfrak{S}_{j}\right)\right\}$. By assumption, there are paths

$$
\psi_{i}:[0, \infty) \rightarrow \phi^{-1}\left(X_{e}(\delta)\right)
$$

such that $\lim _{t \rightarrow \infty} \Phi_{\mathfrak{p}} \circ \phi_{\mathfrak{p}} \circ \psi_{i}(t)=\left\{\mathfrak{S}_{i}\right\}$. Pick $t_{i}>0$ such that

$$
\bigcup \Phi_{\mathfrak{p}} \circ \phi_{\mathfrak{p}} \circ \psi_{i}\left(\left[t_{i}, \infty\right)\right) \subseteq B_{\mathfrak{S}_{i}}\left(\frac{\alpha}{2}\right) \quad \text { for all } 0 \leq i \leq c_{\mathfrak{p}}+1 \text {. }
$$

We will need a more uniform choice for the $t_{i}$ to allow us to apply Lemma 3.6, so we let

$$
R=\max \left\{\frac{8 \kappa}{\alpha \delta^{2}}, d\left(\psi_{1}\left(t_{1}\right), 0\right), d\left(\psi_{2}\left(t_{2}\right), 0\right), \ldots, d\left(\psi_{c_{\mathfrak{p}+1}}\left(t_{c_{\mathfrak{p}+1}}\right), 0\right)\right\} .
$$

Then we take $t_{i}^{\prime}>0$ such that $d\left(\psi_{i}\left(t_{i}^{\prime}\right), e\right)=R$ for all $0 \leq i \leq c_{\mathfrak{p}}+1$.

By our choice of $\alpha$,

$$
B_{\mathfrak{S}_{i}}\left(\frac{\alpha}{2}\right) \cap B_{\mathfrak{S}_{j}}\left(\frac{\alpha}{2}\right)=\varnothing \quad \text { for } i \neq j
$$

Therefore, by (4),

$$
B_{\mathfrak{Z}_{i}}\left(\frac{\alpha}{2}\right) \cap B_{\mathfrak{Z}_{j}}\left(\frac{\alpha}{2}\right)=\varnothing \quad \text { for } i \neq j,
$$

where $\mathfrak{Z}_{i} \in \widehat{X}_{\mathfrak{p}}$ is a sector containing $\phi_{\mathfrak{p}} \circ \psi_{i}\left(t_{i}^{\prime}\right)$. In particular, $\mathfrak{Z}_{i} \notin B_{\mathfrak{Z}_{j}}(\alpha / 2)$ for $i \neq j$. However, we can apply Lemma 3.6 to obtain a proper subset $P$ of $\left\{1, \ldots, c_{\mathfrak{p}}+1\right\}$ such that

$$
\left\{\mathfrak{Z}_{i}\right\}_{i=1}^{c_{\mathfrak{c}}+1} \subseteq \bigcup_{i \in P} B_{\mathfrak{Z}_{i}}\left(\frac{\alpha}{2}\right)
$$

This is a contradiction.

If we assume $\left|\left\{\mathfrak{C}_{i}\right\}_{i=1}^{c_{\infty} \mathfrak{c}_{\mathfrak{p}}+1}\right|>c_{\infty}$, we can arrive at a similar contradiction using Lemma 3.7. The details are carried out in Proposition 5.2 in [3]. 


\section{Independence of basepoint}

So far we have limited ourselves by considering a fixed basepoint $e$. The proof of Theorem 1.2 will require us to hop around from point to point in our quasiflat with holes, and to treat several points as basepoints for the nondegenerate space and, hence, for the limit set of $\phi$. We will need to know therefore, that all of the corresponding nondegenerate spaces and limit sets are compatible with each other-that they are the same up to minor modifications of $\delta$.

The following lemma is essentially Lemma 5.3 from [3].

Lemma 4.1 Let $r>0$ be given and let $e^{\prime} \in X$ be such that $d\left(e, e^{\prime}\right) \leq r$. If $x \in$ $\phi^{-1}\left(X_{e}(\delta)\right)$ and $d(x, 0) \geq \max \{\rho,(6 \kappa r) / \delta\}$ for some $x \in \mathbb{E}^{m}$, then $x \in$ $\phi^{-1}\left(X_{e^{\prime}}(\delta / 2)\right)$ as long as $\delta \leq 1 / 3$.

The next lemma is a short technical remark used in the final lemma of this section.

Lemma 4.2 There exists a constant $v_{4}=v_{4}\left(X_{\mathfrak{p}}\right)$ such that if $\mathfrak{S} \subseteq X_{\mathfrak{p}}$ is a sector based at $e$, and $\mathfrak{S}^{\prime} \subseteq X_{\mathfrak{p}}$ is a sector based at $e^{\prime} \in X_{\mathfrak{p}}$ with $\operatorname{Hd}\left(\mathfrak{S}, \mathfrak{S}^{\prime}\right)<\infty$, then there is a sector $\mathfrak{Z} \subseteq \mathfrak{S} \cap \mathfrak{S}^{\prime}$ such that $\operatorname{Hd}(\mathfrak{Z}, \mathfrak{S}) \leq v_{4} d\left(e, e^{\prime}\right)$.

Proof Let $\mathfrak{S}$ be contained in an apartment $\mathcal{A}$. Then there are isometries $a, n_{1}, n_{2}$, $\ldots, n_{k} \in \operatorname{Isom}\left(X_{\mathfrak{p}}\right)$ such that $a$ stabilizes $\mathcal{A}$, each $n_{i}$ stabilizes a half-space of $\mathcal{A}$ containing a subsector of $\mathfrak{S}^{\prime}$, and $k$ is bounded by a constant depending only on $X$.

It is clear that the result holds if $\mathfrak{S}^{\prime}=a \mathfrak{S}$ or $\mathfrak{S}^{\prime}=n_{i} \mathfrak{S}$. Hence the result for the general $\mathfrak{S}^{\prime}$ holds by the triangle inequality.

We are prepared to show that the $\delta$-limit set of $\phi$ is as independent of the choice of basepoint as one would expect. First though we need to identify the boundaries of $X_{\mathfrak{p}}$ created using two different basepoints. Previously we had defined $\hat{X}_{\mathfrak{p}}$ in a way that depended on $e_{\mathfrak{p}}$. This was done mostly for notational convenience, but the dependence on a basepoint would now be a hindrance for us.

Our solution is to give an equivalent definition of $\hat{X}_{\mathfrak{p}}$ as the space of all sectors with arbitrary basepoints modulo the equivalence that two sectors be identified if they are a finite Hausdorff distance from each other (this is equivalent to the condition that the intersection of the two sectors contains a third sector). Now the metric on $\hat{X}_{\mathfrak{p}}$ is determined by a choice of a basepoint (only up to a Lipschitz equivalence though), but the space $\widehat{X}_{\mathfrak{p}}$ itself is independent of that choice. 
Lemma 4.3 Let $e^{\prime}=\phi\left(0^{\prime}\right)$ for some $0^{\prime} \in \mathbb{E}^{m}$, and suppose $\phi$ is a $(\kappa, \rho, \varepsilon)$ (RGQIE) based at $0^{\prime}$ as well as at 0 . If $\delta \ll 1$, then $\mathcal{L}_{\phi, e^{\prime}}(\delta) \subseteq \mathcal{L}_{\phi, e}(\delta / 2)$.

Proof Suppose $\left(\mathfrak{C}^{\prime}, \mathfrak{S}^{\prime}\right) \in \mathcal{L}_{\phi, e^{\prime}}(\delta)$. Then there is a path $\psi:[0, \infty) \rightarrow \phi^{-1}\left(X_{e^{\prime}}(\delta)\right)$ such that the path $\phi_{\mathfrak{p}} \circ \psi:[0, \infty) \rightarrow X_{\pi_{\mathfrak{p}}\left(e^{\prime}\right)}(\delta)$ escapes every compact set and limits to $\left\{\mathfrak{S}^{\prime}\right\}$ when observed from $\pi_{\mathfrak{p}}\left(e^{\prime}\right)$.

Let $\mathfrak{S}$ be the sector based at $e_{\mathfrak{p}}$ such that $\operatorname{Hd}\left(\mathfrak{S}^{\prime}, \mathfrak{S}\right)<\infty$. Our goal is to show that $\phi_{\mathfrak{p}} \circ \psi$ limits to $\mathfrak{S}$ when observed from $e_{\mathfrak{p}}$.

To this end, for a given $t>0$, let $\mathfrak{S}_{t}$ be a sector based at $e_{\mathfrak{p}}$ such that $\phi_{\mathfrak{p}} \circ \psi(t) \in \mathfrak{S}_{t}$. Let $\mathfrak{S}_{t}^{\prime}$ be a sector based at $\pi_{\mathfrak{p}}\left(e^{\prime}\right)$ such that $\operatorname{Hd}\left(\mathfrak{S}_{t}^{\prime}, \mathfrak{S}_{t}\right)<\infty$. Note that, by Lemma $4.2, \phi_{\mathfrak{p}} \circ \psi(t) \in \mathfrak{S}_{t}^{\prime}$ for sufficiently large values of $t$. Hence, the family $\mathfrak{S}_{t}^{\prime}$ limits to $\mathfrak{S}^{\prime}$ from the vantage point of $\pi_{\mathfrak{p}}\left(e^{\prime}\right)$.

Therefore, for any number $r>0$ and sufficiently large values of $t$, we have $\gamma_{\mathfrak{S}^{\prime}}(r) \in \mathfrak{S}_{t}^{\prime}$. Recall that $\gamma_{\mathfrak{S}^{\prime}}$ is the geodesic ray in $\mathfrak{S}^{\prime}$ based at $\pi_{\mathfrak{p}}\left(e^{\prime}\right)$ that travels down the center of $\mathfrak{S}^{\prime}$ and is used for measuring distances between points in $\hat{X}_{\mathfrak{p}}$ from the vantage point of $\pi_{\mathfrak{p}}\left(e^{\prime}\right)$.

By Lemmas 4.1 and $4.2, \gamma_{\mathfrak{S}^{\prime}}(r) \in \mathfrak{S}_{t}(\delta / 2) \cap \mathfrak{S}(\delta / 2)$. Now applying the no shifting Lemma gives us that

$$
\widehat{d}_{\mathfrak{p}}\left(\mathfrak{S}_{t}, \mathfrak{S}\right) \rightarrow 0
$$

as $t \rightarrow \infty$. Therefore,

$$
\lim _{t \rightarrow \infty} \Phi_{\mathfrak{p}} \circ \phi_{\mathfrak{p}} \circ \psi(t)=\{\mathfrak{S}\}
$$

as desired.

For the symmetric space part of the proof, see Lemma 5.4 of [3].

\section{Proof of Theorem 1.2}

Using the tools we have assembled thus far (in particular large-scale homology of pinched sets, the no shifting Lemma, extending deep points to limit points, the bound on limit points, and the independence of basepoints) we can retrace the proof of Eskin-Farb given in [3] to prove the quasiflats with holes theorem. Since this proof is essentially contained in [3], we will at times only sketch the arguments.

Proof of Theorem 1.2 Since $\Omega_{\left(\varepsilon, \rho^{\prime}\right)} \subseteq \Omega_{(\varepsilon, \rho)}$ when $\rho^{\prime}<\rho$, we may assume that $1 \ll_{(C)} \rho$. We let $\varepsilon$ and $\delta$ be positive numbers such that $\varepsilon \ll \delta \ll 1$. 
As in the proof of Theorem 8.1 of [3], if $x \in \Omega_{(\varepsilon, \rho)}$, we can use a connect-the-dots construction to define a continuous map $\phi_{x}: \mathbb{E}^{m} \rightarrow X$ such that $d\left(\phi_{x}(y), \varphi(y)\right) \leq$ $O(\varepsilon) D_{x}(\rho ; y)$. Hence, $\phi_{x}$ is a $(\kappa, \rho, O(\varepsilon))$ (RGQIE) based at $x$.

Let $\partial X$ be the Tits building for $X$. Because $\hat{X}$ can be identified with the simplices of maximal dimension in $\partial X$, we can measure their distances under the Tits metric. It is well known that if a pair of points in $\hat{X}$ have maximal Tits distance ("opposite points"), then there is a unique flat that contains the pair up to Hausdorff equivalence. Let $F_{1}, \ldots, F_{M}$ be the flats so obtained from pairs of opposite points in $\mathcal{L}_{\phi_{x}, \phi_{x}(x)}(\delta)$. Note that $M \leq\left(c_{\infty} c_{\mathfrak{p}}\right)^{2}$ where $c_{\infty}$ and $c_{\mathfrak{p}}$ are as in Lemmas 3.6 and 3.7.

We will show that $\phi_{x}(x)$ is contained in a bounded neighborhood of $\cup_{i=1}^{M} F_{i}$, but first we want to demonstrate that the limit set, and hence our choice of flats, is independent of $x$.

Suppose $z \in \Omega_{(\varepsilon, \rho)}$ and $\phi_{z}$ is constructed as $\phi_{x}$ to be a $(\kappa, \rho, O(\varepsilon))$ (RGQIE) of $\mathbb{E}^{m}$ based at $z$.

By construction, we have for any point $y \in X$ :

$$
d\left(\phi_{z}(y), \phi_{x}(y)\right) \leq O(\varepsilon)\left(D_{z}(\rho ; y)+D_{x}(\rho ; y)\right) .
$$

It follows that $\phi_{z}$ is a $(2 \kappa, \rho+2 d(x, z), O(\varepsilon))$ (RGQIE) based at $x$. Hence, we obtain through Lemma 4.3 that

$$
\mathcal{L}_{\phi_{z}, \phi_{z}(z)}(\delta) \subseteq \mathcal{L}_{\phi_{z}, \phi_{z}(x)}(\delta / 2) .
$$

If $(\mathfrak{C}, \mathfrak{S}) \in \mathcal{L}_{\phi_{z}, \phi_{z}(x)}(\delta / 2)$, then there is a corresponding limit path $\psi:[0, \infty) \rightarrow$ $\phi_{z}^{-1}\left(X_{\phi_{z}(x)}(\delta / 2)\right)$ that limits to $(\mathfrak{C}, \mathfrak{S})$.

It follows from (5) that $\psi(t) \in \phi_{x}^{-1}\left(X_{\phi_{x}(x)}(\delta / 4)\right)$ for sufficiently large values of $t$.

By projecting $\psi$ onto factors and applying Lemma 3.2 of this paper and Lemma 4.1.i of [3] respectively, we see that

$$
\Theta_{\mathfrak{p}, \phi_{x}(x)}\left(\pi_{\mathfrak{p}} \circ \phi_{x} \circ \psi(t), \pi_{\mathfrak{p}} \circ \phi_{z} \circ \psi(t)\right) \rightarrow 0
$$

and

$$
\Theta_{\infty, \phi_{x}(x)}\left(\pi_{\infty} \circ \phi_{x} \circ \psi(t), \pi_{\infty} \circ \phi_{z} \circ \psi(t)\right) \rightarrow 0
$$

as $t \rightarrow \infty$. The function $\Theta_{\mathfrak{p}, \phi_{x}(x)}$ above is defined analogously to $\Theta_{\mathfrak{p}}$ with a basepoint of $\pi_{\mathfrak{p}}\left(\phi_{x}(x)\right)$ rather than $e_{\mathfrak{p}}$, and $\Theta_{\infty, \phi_{x}(x)}$ is the Furstenberg angle between points in $X_{\infty}$ measured at the point $\pi_{\infty}\left(\phi_{x}(x)\right)$.

Therefore, it must be that $(\mathfrak{C}, \mathfrak{S}) \in \mathcal{L}_{\phi_{x}, \phi_{x}(x)}(\delta / 4)$, and hence,

$$
\mathcal{L}_{\phi_{z}, \phi_{z}(x)}(\delta / 2) \subseteq \mathcal{L}_{\phi_{x}, \phi_{x}(x)}(\delta / 4) .
$$


Joining this inclusion with the previous inclusion of limit sets we have

$$
\mathcal{L}_{\phi_{z}, \phi_{z}(z)}(\delta) \subseteq \mathcal{L}_{\phi_{x}, \phi_{x}(x)}(\delta / 4) .
$$

This shows that our choice of flats is well defined up to replacing $\delta$ with $\delta / 4$.

Now we return to the task of showing that $\phi_{x}(x)$ is within a bounded distance of $\cup_{i=1}^{M} F_{i}$. For the remainder of the proof we let $\phi=\phi_{x}$.

For a fixed $1 \ll_{(\rho, \delta, \varepsilon)} R$ there must be a $y \in \phi^{-1}\left(X_{\phi(x)}(\delta)\right)$ such that $d(\phi(y), \phi(x))=$ $2 R$. Otherwise, we could apply Lemma 2.8 with $W \subseteq \mathbb{E}^{m}$ equal to the sphere centered at $x$ with radius $d(x, y)$ to obtain a contradiction.

Let $e=\left(e_{\infty}, e_{\mathfrak{p}}\right)$ be the midpoint of the geodesic between $\phi(y)$ and $\phi(x)$ so that $\phi(x), \phi(y) \in X_{e}(\delta)$. We project to each factor. Again we will examine the case of a building.

By Proposition 3.5, there are limit points $\left(\mathfrak{C}_{i}, \mathfrak{S}_{i}\right) \in \mathcal{L}_{\phi, e}(\delta)$ for $i=1,2$ such that $\widehat{d}_{\mathfrak{p}}\left(\mathfrak{S}_{1}, \Phi_{\mathfrak{p}} \circ \phi_{\mathfrak{p}}(x)\right) \leq 2 /(\delta R)$ and $\widehat{d}_{\mathfrak{p}}\left(\mathfrak{S}_{2}, \Phi_{\mathfrak{p}} \circ \phi_{\mathfrak{p}}(y)\right) \leq 2 /(\delta R)$. This implies that in the link at $e_{\mathfrak{p}}$-denoted by $L_{e_{\mathfrak{p}}} \subseteq X_{\mathfrak{p}}$-the chambers $\mathfrak{S}_{1} \cap L_{e_{\mathfrak{p}}}$ and $\mathfrak{S}_{2} \cap L_{e_{\mathfrak{p}}}$ are opposite. Therefore, $\mathfrak{S}_{1}$ and $\mathfrak{S}_{2}$ are opposite in $\hat{X}_{\mathfrak{p}}$ under the Tits metric, and there is a unique apartment $\mathcal{A}^{12} \subseteq X_{\mathfrak{p}}$ that contains subsectors of $\mathfrak{S}_{1}$ and $\mathfrak{S}_{2}$.

We also note that the geodesic segments $\left[e_{\mathfrak{p}}, \phi_{\mathfrak{p}}(x)\right]$ and $\left[e_{\mathfrak{p}}, \phi_{\mathfrak{p}}(y)\right]$ can be extended to geodesic rays $\gamma_{x} \subseteq \mathfrak{S}_{1}$ and $\gamma_{y} \subseteq \mathfrak{S}_{2}$ respectively. The bi-infinite path $\gamma_{x} \cup \gamma_{y}$ is a local geodesic, so it is a global geodesic which we name $\gamma$.

As $\gamma$ is a convex subset of Euclidean space, it is contained in an apartment $\mathcal{A}^{\prime} \subseteq X_{\mathfrak{p}}$. Since $\gamma \subseteq \mathcal{A}^{\prime}$, we have that $\mathcal{A}^{\prime}$ contains subsectors of $\mathfrak{S}_{1}$ and $\mathfrak{S}_{2}$. Hence,

$$
e_{\mathfrak{p}} \in \gamma \subseteq \mathcal{A}^{\prime}=\mathcal{A}^{12}
$$

Therefore,

$$
d\left(\phi_{\mathfrak{p}}(x), \mathcal{A}^{12}\right) \leq d\left(\phi_{\mathfrak{p}}(x), e_{\mathfrak{p}}\right) \leq R .
$$

In the proof of Theorem 1.1 of [3], it is shown that there is a constant $\Lambda$, depending only on $X_{\infty}$, and a flat $F^{12} \subseteq X_{\infty}$ that contains $\mathfrak{C}_{1}$ and $\mathfrak{C}_{2}$ up to Hausdorff equivalence, and such that

$$
d\left(\phi_{\infty}(x), F^{12}\right) \leq \frac{1}{2}(\kappa R+C)+\Lambda .
$$

Combining this inequality with its building analogue above yields:

$$
d\left(\phi(x), F^{12} \times \mathcal{A}^{12}\right) \leq \sqrt{R^{2}+\left(\frac{1}{2}(\kappa R+C)+\Lambda\right)^{2}} .
$$

Algebraic $8 \mathcal{G}$ Geometric Topology, Volume 6 (2006) 
The proof of Theorem 1.2 is completed by observing that $F^{12} \times \mathcal{A}^{12} \subseteq X$ is the unique flat that contains $\left(\mathfrak{C}_{1}, \mathfrak{S}_{1}\right)$ and $\left(\mathfrak{C}_{2}, \mathfrak{S}_{2}\right)$ up to Hausdorff equivalence. Hence, $F^{12} \times \mathcal{A}^{12} \in\left\{F_{i}\right\}_{i=1}^{M}$. We take the constant $N$ in the statement of Theorem 1.2 to be $\sqrt{R^{2}+(1 / 2(\kappa R+C)+\Lambda)^{2}}$.

\section{References}

[1] K S Brown, Buildings, Springer, New York (1989) MR969123

[2] A Eskin, Quasi-isometric rigidity of nonuniform lattices in higher rank symmetric spaces, J. Amer. Math. Soc. 11 (1998) 321-361 MR1475886

[3] A Eskin, B Farb, Quasi-flats and rigidity in higher rank symmetric spaces, J. Amer. Math. Soc. 10 (1997) 653-692 MR1434399

[4] A Eskin, B Farb, Quasi-flats in $H^{2} \times H^{2}$, from: "Lie groups and ergodic theory (Mumbai, 1996)”, Tata Inst. Fund. Res. Stud. Math. 14, Tata Inst. Fund. Res., Bombay (1998) 75-103 MR1699359

[5] B Kleiner, B Leeb, Rigidity of quasi-isometries for symmetric spaces and Euclidean buildings, Inst. Hautes Études Sci. Publ. Math. (1997) 115-197 (1998) MR1608566

[6] H M Morse, A fundamental class of geodesics on any closed surface of genus greater than one, Trans. Amer. Math. Soc. 26 (1924) 25-60 MR1501263

[7] G D Mostow, Strong rigidity of locally symmetric spaces, Princeton University Press, Princeton, N.J. (1973) MR0385004

[8] P Pansu, Métriques de Carnot-Carathéodory et quasiisométries des espaces symétriques de rang un, Ann. of Math. (2) 129 (1989) 1-60 MR979599

[9] K Wortman, Quasi-isometric rigidity of higher rank $S$-arithmetic lattices, preprint

[10] K Wortman, Quasi-isometries of rank one $S$-arithmetic lattices, preprint

Department of Mathematics, Yale University, 10 Hillhouse Ave

PO Box 208283, New Haven CT 06520-8283, USA

kevin. wortman@yale.edu

Received: 18 November 2004 